\title{
Estrategia para coordinar las competencias transversales en ingeniería nuclear
}

\author{
Sergio Gallardo ${ }^{\mathrm{a}}$, José Felipe Villanueva ${ }^{\mathrm{b}}$, Sofía Carlos ${ }^{\mathrm{c}}$, Sebastián Martorell ${ }^{\mathrm{d}}$ y Ana \\ Isabel Sánchez ${ }^{\mathrm{e}}$
}

${ }^{a}$ Dpto. de Ingeniería Química y Nuclear, Universitat Politècnica de València, Spain, email: sergalbe@iqn.upv.es; 'Dpto. de Ingeniería Química y Nuclear, Universitat Politècnica de València, Spain, email: jovillo0@upvnet.upv.es; 'DDto. de Ingeniería Química y Nuclear, Universitat Politècnica de València, Spain, email: scarlos@iqn.upv.es; ${ }^{\mathrm{d}}$ Dpto. de Ingeniería Química y Nuclear, Universitat Politècnica de València, Spain, email: smartore@iqn.upv.es; ${ }^{e}$ Dpto. de Estadística e Investigación Operativa Aplicadas y Calidad, Universitat Politècnica de València, Spain, email: aisanche@eio.upv.es

\begin{abstract}
The current curricula of the European Higher Education Area (EHEA) include a series of transversal and specific competences that students must acquire at the end of the different degrees. Through the accreditation process Accreditation Board for Engineering and Technology (ABET), carried out in some degree programs at the Universitat Politècnica de València (UPV), the need to evaluate and obtain evidence of competences, mainly transversal, has been highlighted. Coordination is essential to ensure this point. However, coordination must not remain solely at the level of the entire degree (bachelor or master), but must descend to the level of the subjects. A very specific case is that related to the nuclear engineering field. The purpose of this work is to develop a methodology that systematizes the conceptual coordination of the design of activities, establishment of indicators and coherence of the evaluation of different transversal competences. In the "Results" section, the coordination of the transversal competency "Knowledge of Contemporary Problems" is shown.
\end{abstract}

Keywords: Transversal competencies, nuclear engineering, assessment, indicators.

\footnotetext{
Resumen

Los actuales planes de estudio del Espacio Europeo de Educación Superior (EEES) incluyen una serie de competencias transversales y específicas que deben adquirir los alumnos al finalizar las distintas titulaciones. A través del proceso de acreditación "Accreditation Board for Engineering and Technology" (ABET), llevado a cabo en algunas titulaciones en la Universitat Politècnica de València (UPV), ha quedado patente la necesidad de evaluar y de obtener evidencias de las competencias, principalmente transversales, que deben trabajarse en las titulaciones en cuestión. La coordinación es esencial para poder asegurar este punto. Sin embargo, la
} 
coordinación no debe quedarse únicamente en el nivel de toda la titulación, bien sea grado o máster, sino que debe descender al nivel de las materias. Un caso muy concreto es el relativo a las asignaturas del ámbito de tecnología nuclear. La finalidad de este trabajo es desarrollar una metodología que sistematice la coordinación conceptual del diseño de actividades, establecimiento de indicadores y coherencia de la evaluación de diferentes competencias transversales. En el apartado de "Resultados" se muestra cómo se realizado la coordinación en la competencia "Conocimiento de problemas contemporáneos".

Palabras clave: Competencias transversales, ingeniería nuclear, evaluación, indicadores.

\section{Introducción}

El Espacio Europeo de Educación Superior (EEES) tiene como uno de sus principales objetivos, que los alumnos adquieran una serie de competencias específicas y transversales al finalizar las distintas titulaciones (Sanz de Aceo, 2010). En este contexto queda patente la necesidad explícita de evidenciar la consecución de dichas competencias. Tradicionalmente, el personal docente no ha tenido ningún problema al establecer, trabajar, evaluar y evidenciar la consecución de las competencias específicas por asignaturas y materias en la titulación. Este proceso está completamente integrado en la forma de trabajo de los docentes y tiene, en general, una estrecha relación con la faceta investigadora de cada uno. Sin embargo, el establecimiento, aplicación y evaluación de las competencias transversales ha quedado, en términos generales, relegado a un segundo plano hasta ahora (Villa et al, 2007). En este sentido, la Universitat Politècnica de València, a través del proyecto institucional "Competencias Transversales UPV" (UPV, 2015) ha definido dos objetivos principales: a) Establecer una estrategia de evaluación sistemática de las competencias transversales, definiendo dónde se adquieren y cómo deben ser evaluadas y b) Acreditar la adquisición de dichas competencias. Los beneficios derivados del éxito de este proyecto son: a) Proporcionar a sus egresados un valor añadido diferenciador de otros egresados, b) Poner en valor la capacitación de los egresados de cara a los empleadores y c) Explicitar la adquisición de las competencias para acreditaciones internacionales. Garantizar la adquisición de las competencias no es un hecho trivial. Por ello, el proyecto institucional de la UPV propone tres vías para ello: las titulaciones, el Trabajo Fin de Grado y/o Trabajo Fin de Máster (TFG/TFM) y actividades extracurriculares (trabajo en departamentos, institutos, empresas, organizaciones, cursos de formación posgrado, etc.). Además, establece tres niveles de dominio para aumentar progresivamente el grado de profundización del alumnado en cada competencia. La UPV ha establecido 13 competencias que caracterizan globalmente el perfil competencial del egresado. Las Escuelas y Facultades han realizado una importante labor de coordinación entre materias y entre asignaturas para asegurar que todas las competencias se trabajan en los diferentes niveles de dominio a través de las tres vías mencionadas. Descendiendo al nivel de las asignaturas, resulta evidente la necesidad de establecer grupos de trabajo entre asignaturas

(cc) EY-NC-ND 2018, Universitat Politècnica de València

Congreso IN-RED (2018) 
afines para que los profesores establezcan criterios uniformes para la definición de parámetros de trabajo, evaluación y evidenciación de las competencias (UPV, 2015b). La coordinación es esencial para poder asegurar este punto. Un caso muy concreto es el relativo a las asignaturas del ámbito de ingeniería nuclear. La especificidad de contenidos obliga a redoblar los esfuerzos de coordinación para evitar solapes y asegurar un diseño, desarrollo y evaluación coherente de las competencias transversales y específicas (Gallardo et al., 2015 y Villanueva et al., 2016). La coordinación exige realizar un ejercicio de síntesis de contenidos y mostrar de forma explícita la integración de las distintas asignaturas, en este caso del campo de conocimiento de nuclear.

\section{Objetivos}

El objetivo principal de este trabajo es establecer una metodología que permita sistematizar las actividades y tareas relacionadas con la coordinación de competencias transversales en asignaturas del ámbito de la ingeniería nuclear. Con este fin se establecen los siguientes objetivos específicos:

- Puesta en común del significado de cada una de las competencias transversales para los profesores involucrados. Alcanzar una definición consensuada de cada competencia.

- Consensuar el perfil competencial del alumno egresado en materias de ingeniería nuclear.

- Obtención de rúbricas en función de la competencia, vía y nivel de dominio. Establecimiento de indicadores comunes entre asignaturas.

- Realimentación a los profesores respecto del grado de implantación y resultados de la metodología.

\section{Desarrollo de la innovación}

\subsection{Las asignaturas}

En los últimos años, se ha detectado una falta de coordinación en materia de competencias transversales en las asignaturas que se imparten en el Dpto de Ingeniería Química y Nuclear de la Universitat Politècnica de València. El presente trabajo se centra en las asignaturas que imparte la Unidad Docente (UD) de Ingeniería Nuclear. Dicha Unidad imparte docencia en los siguientes Grados: Ingeniería en Tecnologías Industriales (GITI), Ingeniero de la Energía (GIE), Ingeniería en Organización Industrial (GIOI), Ingeniería Química (GIQ) e Ingeniería Biomédica (GIB). Además, se imparte docencia en el Máster Universitario (MU) de Ingeniería Industrial, $\mathrm{MU}$ en Seguridad Industrial y Medio Ambiente y en el MU en Tecnología Energética para el Desarrollo Sostenible. La UD de Ingeniería Nuclear imparte docencia en asignaturas relacionadas con la generación y el uso de la energía (energías renovables, energía nuclear, termohidráulica, aplicaciones de las radiaciones ionizantes, etc.). En este contexto, con docencia en múltiples grados y másteres es difícil realizar una coordinación a nivel global, por lo que se ha propuesto comenzar por 
una coordinación en el Grado de Ingeniero de la Energía, ya que es el Grado con mayor número de asignaturas en las que imparte docencia Ingeniería Nuclear:

- Energía y Desarrollo Sostenible: curso $2^{\circ}$ B. Asignatura Obligatoria. Número de créditos: 6

- Tecnología Nuclear: curso $4^{\circ} \mathrm{A}$. Asignatura Obligatoria. Número de créditos: 6

- Centrales Nucleares Avanzadas: curso $4^{\circ}$ B. Asignatura Optativa. Número de créditos: 4,5

- Seguridad Nuclear: curso $4^{\circ}$ B. Asignatura Optativa. Número de créditos: 4,5

- Operación de Reactores Nucleares: curso $4^{\circ}$ B. Asignatura Optativa. Número de créditos: 4,5

- Protección Radiológica: curso $4^{\circ}$ B. Asignatura Optativa. Número de créditos: 4,5

La asignatura "Energía y Desarrollo Sostenible" es introductoria y totalmente descriptiva, mientras que las asignaturas de $4^{\circ}$ curso son muy específicas y de marcado carácter nuclear. Muchas de las asignaturas de $4^{\circ}$ pertenecen a la optatividad y cuentan con un reducido número de alumnos.

\subsection{Las competencias transversales}

Las asignaturas mencionadas en el apartado anterior son punto de control de diferentes competencias, tal y como se muestra en la Tabla 1. Las competencias con mayor peso en estas asignaturas son: Comprensión e integración; Aplicación y pensamiento práctico; Responsabilidad ética, medioambiental y profesional; Conocimiento de problemas contemporáneos e Instrumental específica.

Tabla 1. Distribución de competencias transversales por asignaturas.

\begin{tabular}{|c|c|c|c|c|c|c|c|c|c|c|c|c|c|}
\hline & 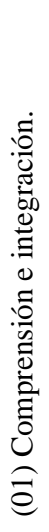 & 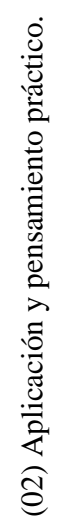 & 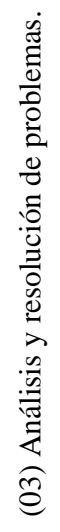 & 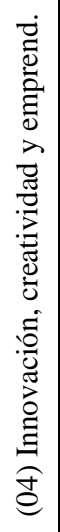 & 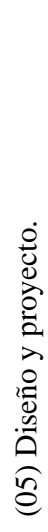 & 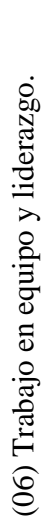 & 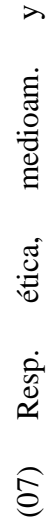 & 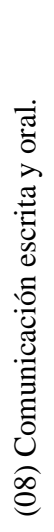 & 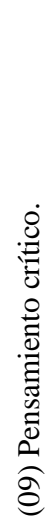 & 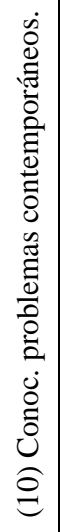 & 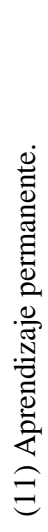 & 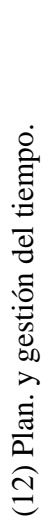 & 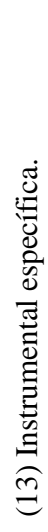 \\
\hline Energía y Desarrollo Sostenible & & & & & & & $\mathrm{x}$ & & & $\mathrm{x}$ & & & $\mathrm{x}$ \\
\hline Tecnología Nuclear & & $\mathrm{x}$ & & & & & & & & $\mathrm{x}$ & & & $\mathrm{x}$ \\
\hline Centrales Nucleares Avanzadas & & $\mathrm{x}$ & & & & & & & & $\mathrm{x}$ & & & \\
\hline
\end{tabular}

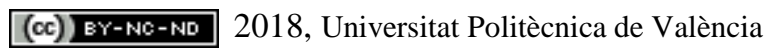

Congreso IN-RED (2018) 


\begin{tabular}{|l|l|l|l|l|l|l|l|l|l|l|l|l|l|}
\hline Seguridad Nuclear & $\mathrm{x}$ & & & & & & & & & $\mathrm{x}$ & & & $\mathrm{x}$ \\
\hline $\begin{array}{l}\text { Operación de Reactores } \\
\text { Nucleares }\end{array}$ & $\mathrm{x}$ & & & & & & & & & & & & $\mathrm{x}$ \\
\hline Protección Radiológica & $\mathrm{x}$ & & & & & & & & & $\mathrm{x}$ & & & $\mathrm{x}$ \\
\hline
\end{tabular}

\subsection{La reunión inicial}

Una vez caracterizado el escenario en cuanto a las asignaturas y competencias, es necesario entablar un debate para dirimir el significado de cada competencia. Esta reunión pretende uniformizar los conceptos de partida, eliminar ideas preconcebidas y prejuicios. Se podría pensar que el propio enunciado de la competencia es suficiente para determinarla, pero el trabajo diario ha demostrado que no todos los profesores conciben la misma competencia de igual forma, en ocasiones por interpretaciones erróneas de la misma o simplemente por desconocimiento. Queda pues patente, que es necesario planificar una primera reunión para acordar su significado. Un claro ejemplo de errores de concepto aparece cuando se trabaja la “Análisis y resolución de problemas”. Algunos profesores piensan erróneamente que esta competencia consiste en plantear y resolver problemas de una determinada asignatura y no en establecer estrategias y metodologías para resolver problemas (de cualquier tipo) de una forma sistematizada. Otro ejemplo, ¿Qué entendemos por "Conocimiento de Problemas Contemporáneos”? ¿En qué se diferencia un problema contemporáneo de cualquier otro problema? A priori, son preguntas que parecen tener fácil respuesta hasta que se realiza un análisis en profundidad. La reunión se planifica en el calendario académico antes de publicar las Guías Docentes y debe servir para establecer las bases sobre las que se va a construir toda la estructura docente. Para ayudar a consensuar los conceptos que se van a utilizar, se ha realizado una búsqueda en el Portal de Buenas Prácticas Docentes el Ministerio de Educación, Ciencia y Deporte, para localizar experiencias previas en el ámbito del trabajo en competencias transversales. Se ha encontrado diverso material que se puede categorizar en 7 Recursos de Desarrollo, 14 Recursos de Estudio, 27 Recursos de Experiencia, 5 Recursos de Innovación y 6 Recursos de Investigación. Respecto de los trabajos en innovación e investigación, destacan los realizados por Rodríguez et al., 2015 y Galván et al, 2015, En las actas de las ediciones de 2015 y 2016 del congreso de innovación docente IN-RED, se pueden encontrar distintos trabajos de innovación que profundizan en diversos aspectos de las competencias transversales, fundamentalmente en la mejora en la adquisición de alguna competencia por parte de los alumnos. Sin embargo, las únicas reseñas que hemos localizado que se centran en el problema de la coordinación de competencias transversales son: Berbegal et al, 2015 y Ochoa et al, 2015. La falta de bibliografía y referencias en este contexto evidencia la necesidad de realizar una experiencia documentada en la coordinación de competencias transversales. 


\subsection{El perfil del egresado}

Las Escuelas y Facultades intentan asegurar que los egresados de los distintos grados y másteres adquirirán capacidades en múltiples aspectos. Esta labor de coordinación a nivel de título es compleja y está sometida a revisiones internas y externas, como las realizadas en el proceso de acreditación "Accreditation Board for Engineering and Technology (ABET)”, o en las acreditaciones que llevan a cabo la ANECA o la AVAP (en la Comunitat Valenciana).

¿Qué capacidades debe tener un egresado que haya cursado las asignaturas de ingeniería nuclear? ¿Cuál debe ser su nivel de dominio? ¿Cómo se conseguirá que ese perfil sea una diferencia cualitativa respecto de otros egresados? Estas preguntas no tienen fácil respuesta.

Se ha realizado un cuestionario para determinar qué competencias serían las más adecuadas en las asignaturas de nuclear y cuál debería ser el nivel de dominio. En términos generales, la conclusión es que los puntos de control asignados por la Escuela a las asignaturas de nuclear son representativos, coherentes y se adecúan a los contenidos y objetivos de las mismas. En cuanto a los niveles de dominio, es lógico que en las asignaturas de primeros cursos el nivel de dominio sea bajo (I) y en $4^{\circ}$ de grado o en máster, más elevado (II y III, respectivamente).

\subsection{Diseño de actividades}

El objetivo es diseñar actividades que se puedan utilizar de base en distintas asignaturas, especialmente las de $4^{\circ}$ curso, tomando como referencia la asignatura obligatoria de $2^{\circ}$ curso ("Energía y Desarrollo Sostenible"). Con esta idea en mente, la coordinación se centra en aportar coherencia a los objetivos, metodología y resultados para diseñar una serie de actividades que puedan trabajarse en las diferentes asignaturas simultáneamente (teniendo en cuenta la formación inicial de los alumnos).

Partiendo de la idea vertebradora de la producción de energía eléctrica mediante nuclear, en la asignatura de $2^{\circ}$ curso de GIE se proponen diferentes actividades relacionadas para familiarizarse con conceptos básicos de la energía nuclear. En este contexto, con el objetivo de trabajar y evaluar diferentes competencias transversales, se proponen a los alumnos textos para debatir y suscitar polémica: la generación de residuos, la fiabilidad de la producción de origen nuclear, los riesgos asociados, etc. En este contexto, el alumnado además de trabajar ciertas competencias específicas, trabajan determinadas competencias transversales. Con estas sencillas actividades, introductorias al campo nuclear, el alumno realiza una primera toma de contacto. Conforme se avanza en los cursos, se incrementa la exigencia en el nivel de trabajo de las CTs. En la asignatura de $2^{\circ}$ curso se trabaja con verbos de nivel bajo según la taxonomía de Bloom (enumerar, identificar, ...), mientras que en las asignaturas de $4^{\circ}$ curso se trabaja con verbos como razonar, sintetizar, argumentar, establecer, obtener, etc.

Una vez que se realiza la puesta en común de las actividades que se realizarán en las diferentes asignaturas, es necesario definir los indicadores mediante los que se evaluará el trabajo en CTs.

(cc) EY-NC-ND 2018, Universitat Politècnica de València

Congreso IN-RED (2018) 


\subsection{Rúbricas de evaluación e indicadores}

Se parte de las propuestas publicadas en el marco del proyecto institucional de competencias transversales UPV y se procede a analizar las rúbricas genéricas de evaluación para adaptarlas a las asignaturas de la Tabla 1 . Se han utilizado entre 2 y 4 indicadores de carácter general para que se puedan adaptar a las distintas asignaturas. En las Tablas 2 a 6 se muestran los indicadores para cada competencia considerada. A partir de estos indicadores semi-genéricos, se desarrollarán los indicadores para cada actividad de cada asignatura.

Tabla 2. Comprensión e integración (nivel II).

\begin{tabular}{|l|l|}
\hline Objetivo & Relacionar e integrar conceptos en situaciones complejas en Ingeniería Nuclear. \\
\hline Indicadores & Establecer relaciones entre diferentes conceptos físicos y tecnológicos. \\
\hline & Integrar elementos de diferentes asignaturas para la interpretación de la realidad. \\
\hline
\end{tabular}

Tabla 3. Aplicación y pensamiento práctico (nivel II).

\begin{tabular}{|l|l|}
\hline Objetivo & Diseñar un plan con acciones concretas para situaciones nuevas. \\
\hline Indicadores & Establecer objetivos concretos en relación con las situaciones que se le plantean. \\
\hline & Obtener la información necesaria para abordar las situaciones. \\
\hline & Sistematizar la elaboración del plan, identificando las etapas. \\
\hline
\end{tabular}

Tabla 4. Responsabilidad ética y profesional y medioambiental (Nivel I).

\begin{tabular}{|c|c|}
\hline Objetivo & Ser conscientes de los valores a partir de los que se construye la sociedad. \\
\hline \multirow[t]{3}{*}{ Indicadores } & $\begin{array}{l}\text { Aceptar críticamente nuevas perspectivas, aunque cuestionen las propias en relación con la } \\
\text { energía nuclear. }\end{array}$ \\
\hline & $\begin{array}{l}\text { Diferenciar hechos y opiniones o interpretaciones. Identificar los fundamentos y reconocer } \\
\text { malinterpretaciones o manipulaciones. }\end{array}$ \\
\hline & Reconocer los conceptos éticos y deontológicos relacionados con la energía nuclear. \\
\hline Objetivo & $\begin{array}{l}\text { Valorar las actuaciones profesionales en cuanto a repercusión social, ambiental y } \\
\text { económica. }\end{array}$ \\
\hline \multirow[t]{3}{*}{ Indicadores } & Comprender el papel de la energía nuclear en la sociedad actual. \\
\hline & $\begin{array}{l}\text { Identificar los pros } \mathrm{y} \text { contras de } \\
\text { medioambientales. }\end{array}$ \\
\hline & Actuar individualmente en armonía con los planes consensuados y vigentes. \\
\hline
\end{tabular}

Tabla 5. Conocimiento de problemas contemporáneos (Nivel II).

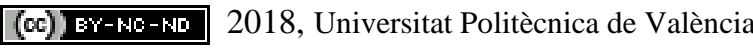

Congreso In-Red (2018) 


\begin{tabular}{|l|l|}
\hline Objetivo & $\begin{array}{l}\text { Analizar los problemas contemporáneos relacionados con la energía nuclear y sus } \\
\text { aplicaciones. }\end{array}$ \\
\hline & $\begin{array}{l}\text { Distinguir las partes que componen un problema, identificando los principales actores en } \\
\text { sus diferentes dimensiones (económica, social, ética, tecnológica...). }\end{array}$ \\
\hline & Razonar las soluciones ya propuestas para un problema. \\
\hline & $\begin{array}{l}\text { Proponer nuevas soluciones al problema que se ha planteado a partir de la propia } \\
\text { experiencia y de la información disponible. }\end{array}$ \\
\hline
\end{tabular}

Tabla 6. Instrumental específica (Nivel II).

\begin{tabular}{|l|l|}
\hline Objetivo & $\begin{array}{l}\text { Integrar correctamente las herramientas básicas del ámbito profesional de forma } \\
\text { autónoma. }\end{array}$ \\
\hline Indicadores & Manejar las herramientas básicas de forma autónoma. \\
\hline & Conocer las limitaciones de las herramientas básicas. \\
\hline
\end{tabular}

En las tablas anteriores se ha tenido en cuenta el nivel de dominio II para la mayoría de las competencias.

Con los indicadores definidos y adaptados a las actividades de las distintas asignaturas, se lleva a cabo la evaluación de las competencias. El proceso finaliza con una retroalimentación de los alumnos a los profesores y de éstos últimos al resto de profesores mediante una reunión de cierre, que establece el inicio de la siguiente interacción. En otras palabras, se lleva a cabo un proceso de mejora continua PDCA - Plan, Do, Check, Act -

\section{Resultados}

En este apartado se muestra, a modo de ejemplo, los resultados correspondientes a la competencia de "Conocimiento de Problemas Contemporáneos" (CT-10). Para el resto de competencias analizadas en la coordinación, se han obtenido resultados análogos que se omiten por motivos de concisión.

\subsection{Resultados de la reunión inicial}

Siguiendo la metodología expuesta en el apartado de "Desarrollo de la Innovación”, en la primera reunión de coordinación se realiza una puesta en común para alcanzar una caracterización consensuada de dicha competencia y del perfil del egresado.

El brainstorming realizado arroja las siguientes ideas:

- Problemas actuales que afectan a gran parte de la sociedad.

- Procesos históricos que, en la actualidad se encuentran en pleno desarrollo.

- Comprenden cuestiones sociales, económicas, medioambientales y normativas.

(cc) EY-NC-ND 2018, Universitat Politècnica de València

Congreso IN-RED (2018) 
- Tienen repercusiones importantes a nivel global.

- Generalmente, de forma indirecta tienen relación con los contenidos de las asignaturas.

- Se pueden estudiar de forma interdisciplinar.

La siguiente pregunta que se plantea en la reunión de puesta en común es, ¿cómo se transmite la importancia de esta competencia a los alumnos? Debemos conseguir:

- que el alumnado se sienta artífice de los momentos históricos que vivimos.

- trasladarles la idea de que conocer el escenario actual económico, social y medioambiental les proporciona una ventaja competitiva respecto de otros egresados.

- que se sientan que su generación "puede hacer las cosas mejor” que las anteriores generaciones.

- Q Que el alumnado sea consciente de cómo se ha llegado a la situación actual.

En definitiva, debemos ser capaces de transmitirles la ilusión por mejorar la sociedad, con todo lo que ello implica. La siguiente pregunta es: ¿cómo podemos lograrlo? Creemos que la respuesta a esta pregunta es mediante actividades que estimulen sus sentidos para que perciban el trasfondo social y humano del problema, y no se queden con la impersonalidad de los modelos, las ecuaciones y los números.

\subsection{Resultados del diseño de actividades}

Con esta idea, el grupo de trabajo ha diseñado diversas actividades para trabajar simultáneamente diferentes competencias. En este caso, se presenta la actividad que se inicia en la asignatura de $2^{\circ}$ curso y que se continúa en la obligatoria y en las optativas de $4^{\circ}$.

Actividad en $2^{\circ}$ curso.

En la asignatura obligatoria “Energía y Desarrollo Sostenible” de $2^{\circ}$ se presenta el tema de nuclear a los alumnos del Grado de Ingeniero de la Energía. El grupo de trabajo ha concluido que la mejor opción para captar la atención de los estudiantes y generar un espacio propicio para el debate es presentar el accidente de la central nuclear de Fukushima Daichi (Japón). Se trata de un suceso complejo que implica todos los aspectos que se estudian a lo largo del grado, por lo que se considera idóneo para suscitar el interés del alumnado. En la actividad se hace una breve descripción del suceso y se proporciona información que debe ser revisada por los alumnos por grupos. Revisada dicha información, el profesor plantea las siguientes afirmaciones (todas ellas falsas):

1. El accidente de Fukushima fue inevitable.

2. El accidente ha supuesto el inicio de la desaparición de las centrales nucleares comerciales.

3. En el escenario actual de España, se puede prescindir de la energía nuclear. 
4. La energía nuclear presenta más inconvenientes que ventajas.

A continuación, unos grupos deben defender estas afirmaciones y otros las deben rebatir, siempre con datos objetivos, evitando las opiniones y desarrollando un discurso coherente. Los grupos nombran un portavoz y se inicia el debate entre los que defienden y los que niegan las afirmaciones.

El debate resultante es muy valioso porque surgen de forma natural interrelaciones del problema contemporáneo de la energía con otros problemas: los residuos nucleares, el impacto medioambiental, la crisis económica, el calentamiento global (emisiones de CO2).

Al final de la sesión se elabora un listado de los argumentos más objetivos y mejor construidos. Se realizó un cuestionario al alumnado que había participado en la actividad para conocer los siguientes puntos:

- $\quad$ ¿Te ha parecido útil esta actividad?

- ¿ ¿Tenías ideas preconcebidas respecto de la energía nuclear antes de estudiar este accidente?

- ¿ ¿Ha cambiado algún aspecto de tu visión del problema de la energía?

- ¿Crees que en la actualidad la sociedad puede prescindir de la energía nuclear? En caso afirmativo, ¿cómo?

- ¿ ¿Cuál ha sido la lección aprendida de este accidente?

- ¿Crees que lo que hemos estudiado en la actividad representa un problema contemporáneo?

Los resultados del cuestionario son muy satisfactorios:

- $\quad$ Aproximadamente el 80\% del alumnado considera útil la actividad;

- $\quad$ El 55\% admite tener ideas preconcebidas;

- $\quad$ El 75\% cree que la actividad les ha permitido ampliar su visión del problema energético;

- $\quad$ El 95\% cree que en la actividad se ha trabajado un problema contemporáneo.

Actividades en $4^{\circ}$ curso.

En la asignatura obligatoria "Tecnología Nuclear" se retoma el tema del accidente de Fukushima y, gracias a los contenidos estudiados y a las competencias específicas trabajadas, se puede reevaluar el accidente y su relación con los problemas contemporáneos desde un nivel de profundidad mayor.

La actividad consiste en evaluar los costes de dicho accidente desde diferentes enfoques:

- Impacto económico. ¿Cómo ha repercutido el accidente a escala mundial en la producción de energía? ¿Ha sido determinantes para que algunos países abandonen el programa nuclear? ¿Y España, podría hacerlo en la actualidad?

(cc) EY-NC-ND 2018, Universitat Politècnica de València

Congreso IN-RED (2018) 
- Impacto medio ambiental. ¿Cómo ha afectado la contaminación radiactiva al entorno? ¿ Ha tenido repercusión mundial? ¿Se llegó a monitorizar en España un incremento de radionucleidos emitidos por la central?

- Impacto humano: ¿Cuáles han sido los efectos sufridos en algunas personas? ¿Cuál será el efecto a largo plazo?

- ¿ ¿De qué manera afectarán estos impactos a otros problemas contemporáneos?

En este caso, debido a la profundidad de las preguntas, la dinámica escogida es la elaboración de un trabajo académico por grupos y su exposición en clase.

En la asignatura optativa de 4 "Protección Radiológica", la actividad propuesta se centra en el cálculo de dosis producido por el accidente y su incidencia en aspectos biológicos. Para ahondar en el trabajo de la competencia transversal objeto de estudio, se propone comparar los efectos de este accidente con otros ocurridos en la historia, fundamentalmente el accidente de Chernobyl y el de Three Mile Island. La comparación en cuanto al escenario, las causas del accidente y las consecuencias, permiten al alumno conocer cómo ha cambiado la cultura de la seguridad en el ámbito de la energía nuclear, siendo este tema un problema contemporáneo que los profesores queremos destacar. Debido al reducido número de alumnos matriculados en la asignatura (entre 5 y 10 en función del curso académico), se considera que una exposición individual de unos 15 minutos es lo más adecuado para evaluar la actividad.

En la asignatura optativa de $4^{\circ}$ "Seguridad Nuclear", se estudia el accidente desde el punto de vista de las salvaguardias tecnológicas, los sistemas de seguridad y las probabilidades de fallo. En este caso, además de cálculos numéricos se propone estudiar cómo se ha endurecido la normativa internacional en materia de seguridad como lección aprendida. La actividad requiere un esfuerzo notable por parte de los alumnos para buscar, ordenar e interpretar cómo afectan las nuevas normativas internacionales a esta materia. Se destaca el problema de seguridad a escala global como un problema contemporáneo y las fortalezas y debilidades de la energía nuclear en este ámbito. La evidencia de la actividad es un informe donde el alumnado describe los cambios de normativa y su repercusión la vida útil de las centrales.

Por último, en "Centrales Nucleares Avanzadas", se estudian posibles escenarios futuros. La pregunta es: ¿Y qué nos espera en los próximos 30 años? ¿Es posible un nuevo Fukushima? ¿Lograrán los nuevos diseños de reactores reducir la probabilidad de accidente a un valor extremadamente pequeño? El enfoque de la actividad es relacionar los diseños de generación III y IV con los actuales diseños e identificar cómo incorporan dichos diseños las lecciones aprendidas de accidentes y fallos anteriores. La actividad es un trabajo académico con exposición en clase. De nuevo, se pone especial atención en señalar cómo se enmarca este concepto en los problemas actuales de la sociedad: el suministro de energía y la seguridad medioambiental y social. 


\subsection{La rúbrica para cada asignatura}

Tal y como se expuesto, en las cinco asignaturas se estudia el mismo caso intentando dar una coherencia y línea de continuidad entre ellas. En este contexto, la evaluación también debe ser coherente y lo más objetiva posible.

Se ha considerado que lo más acertado es adaptar la rúbrica de nivel de dominio I o II a las características de la actividad. A continuación, se muestra la rúbrica de la actividad descrita en el apartado anterior para cada asignatura (Tablas 7 a 11).

Tabla 7. Rúbrica para “Energía y Desarrollo Sostenible” - Nivel de dominio I-

\begin{tabular}{|c|c|c|c|c|}
\hline Indicador & $\begin{array}{ll}\text { D. No } \\
\text { alcanzado }\end{array}$ & C. En desarrollo & $\begin{array}{l}\text { B. Bien } \\
\text { /adecuado }\end{array}$ & $\begin{array}{l}\text { A. Excelente / } \\
\text { Ejemplar }\end{array}$ \\
\hline $\begin{array}{l}\text { Identifica y describe aspectos sociales } \\
\text { se ven afectados por un accidente } \\
\text { nuclear. }\end{array}$ & $\begin{array}{l}\text { No identifica } \\
\text { ningún aspecto } \\
\text { social. }\end{array}$ & $\begin{array}{l}\text { Identifica uno o } \\
\text { dos problemas } \\
\text { sociales. }\end{array}$ & $\begin{array}{l}\text { Identifica y } \\
\text { describe uno o } \\
\text { dos problemas } \\
\text { sociales. }\end{array}$ & $\begin{array}{l}\text { Identifica y } \\
\text { describe más de } \\
\text { dos problemas } \\
\text { sociales. }\end{array}$ \\
\hline $\begin{array}{l}\text { Identifica y describe } \text { aspectos } \\
\text { medioambientales se ven afectados } \\
\text { por un accidente nuclear. }\end{array}$ & $\begin{array}{l}\text { No identifica } \\
\text { ningún medio } \\
\text { ambiental. }\end{array}$ & $\begin{array}{l}\text { Identifica uno o } \\
\text { dos problemas } \\
\text { medio } \\
\text { ambientales. }\end{array}$ & $\begin{array}{l}\text { Identifica y } \\
\text { describe uno o } \\
\text { dos problemas } \\
\text { medio } \\
\text { ambientales. }\end{array}$ & $\begin{array}{l}\text { Identifica y } \\
\text { describe más de } \\
\text { dos problemas } \\
\text { medio } \\
\text { ambientales. }\end{array}$ \\
\hline $\begin{array}{l}\text { Identifica y describe repercusiones } \\
\text { económicas. }\end{array}$ & $\begin{array}{l}\text { No identifica } \\
\text { ninguna } \\
\text { repercusión } \\
\text { económica. }\end{array}$ & $\begin{array}{l}\text { Identifica una o } \\
\text { dos } \\
\text { repercusiones } \\
\text { económicas. }\end{array}$ & $\begin{array}{ll}\text { Identifica } & \text { y } \\
\text { describe una } & \text { o } \\
\text { dos } & \\
\text { repercusiones } & \\
\text { económicas. } & \end{array}$ & $\begin{array}{l}\text { Identifica y } \\
\text { describe más de } \\
\text { dos } \\
\text { repercusiones } \\
\text { económicas. }\end{array}$ \\
\hline
\end{tabular}

Tabla 8. Rúbrica para "Tecnología Nuclear” - Nivel de dominio II-

\begin{tabular}{|l|l|l|l|l|l|}
\hline Indicador No & C. En desarrollo & $\begin{array}{l}\text { B. } \\
\text { /adecuado }\end{array}$ & Bien & $\begin{array}{l}\text { A. Excelente / } \\
\text { Ejemplar }\end{array}$ \\
\hline $\begin{array}{l}\text { Argumenta si el accidente fue } \\
\text { evitable. iexiste el fallo de } \\
\text { probabilidad 0? Razona si hay } \\
\text { prejuicios y desinformación en este } \\
\text { aspecto. }\end{array}$ & No argumenta. & $\begin{array}{l}\text { Argumenta si el } \\
\text { accidente fue } \\
\text { evitable. }\end{array}$ & $\begin{array}{l}\text { Argumenta si el } \\
\text { accidente } \\
\text { evitable }\end{array}$ & $\begin{array}{l}\text { Argumenta si el } \\
\text { accidente } \\
\text { fue } \\
\text { evitable } \\
\text { razona } \\
\text { superficialmente } \\
\text { otros aspectos. } \\
\text { detalladamente } \\
\text { otros aspectos. }\end{array}$ \\
\hline $\begin{array}{l}\text { Establece y describe relaciones entre } \\
\text { el accidente, la tecnología nuclear y } \\
\text { otras formas de producción de } \\
\text { energía. }\end{array}$ & $\begin{array}{l}\text { No establece } \\
\text { ninguna } \\
\text { relación. }\end{array}$ & $\begin{array}{l}\text { Establece } \\
\text { alguna relación } \\
\text { pero no la } \\
\text { describe. }\end{array}$ & $\begin{array}{l}\text { Establece } \\
\text { describe alguna } \\
\text { relación. }\end{array}$ & $\begin{array}{l}\text { Establece } \\
\text { describe más de } \\
\text { tres relaciones. }\end{array}$ \\
\hline
\end{tabular}

Tabla 9. Rúbrica para “Protección Radiológica” - Nivel de dominio II-

\begin{tabular}{|l|l|l|l|l|l|}
\hline Indicador & $\begin{array}{l}\text { D. No } \\
\text { alcanzado }\end{array}$ & C. En desarrollo & $\begin{array}{l}\text { B. } \\
\text { /adecuado }\end{array}$ & $\begin{array}{l}\text { A. Excelente / } \\
\text { Ejemplar }\end{array}$ \\
\hline
\end{tabular}




\begin{tabular}{|l|l|l|l|l|l|}
\hline $\begin{array}{l}\text { Analiza y alcanza conclusiones sobre } \\
\text { los riesgos y beneficios de las } \\
\text { radiaciones ionizantes: medicina, } \\
\text { industria, energía. }\end{array}$ & $\begin{array}{l}\text { No analiza ni } \\
\text { alcanza ninguna } \\
\text { conclusión. }\end{array}$ & $\begin{array}{l}\text { Analiza } \\
\text { someramente } \\
\text { algunos riesgos. }\end{array}$ & $\begin{array}{l}\text { Analiza } \\
\text { alcanza } \\
\text { conclusiones } \\
\text { para algunos } \\
\text { riesgos. }\end{array}$ & $\begin{array}{l}\text { Analiza } \\
\text { alcanza } \\
\text { conclusiones } \\
\text { para } \\
\text { beneficios. }\end{array}$ \\
\hline $\begin{array}{l}\text { Argumenta las consecuencias } \\
\text { biológicas de las radiaciones } \\
\text { ionizantes. Identifica que las } \\
\text { consecuencias no se producen } \\
\text { únicamente por las centrales } \\
\text { nucleares. }\end{array}$ & $\begin{array}{l}\text { No identifica ni } \\
\text { argumenta. }\end{array}$ & $\begin{array}{l}\text { Identifica } \\
\text { algunas } \\
\text { consecuencias } \\
\text { pero no las } \\
\text { analiza. }\end{array}$ & $\begin{array}{l}\text { Identifica } \\
\text { algunas } \\
\text { consecuencias } \\
\text { pero las analiza } \\
\text { superficialmente. }\end{array}$ & $\begin{array}{l}\text { Identifica } \\
\text { analiza } \\
\text { consecuencias } \\
\text { razonadamente. }\end{array}$ \\
\end{tabular}

Tabla 10. Rúbrica para "Seguridad nuclear” - Nivel de dominio II-

\begin{tabular}{|c|c|c|c|c|}
\hline Indicador & $\begin{array}{ll}\text { D. } & \text { No } \\
\text { alcanzado } & \end{array}$ & C. En desarrollo & $\begin{array}{ll}\text { B. } & \text { Bien } \\
\text { /adecuado } & \end{array}$ & $\begin{array}{l}\text { A. Excelente / } \\
\text { Ejemplar }\end{array}$ \\
\hline $\begin{array}{l}\text { Analiza aspectos humanos y técnicos } \\
\text { de la seguridad. Establece relaciones } \\
\text { con ámbitos sociales y } \\
\text { medioambientales. }\end{array}$ & $\begin{array}{l}\text { No analiza ni } \\
\text { establece } \\
\text { relaciones. }\end{array}$ & $\begin{array}{l}\text { Analiza algún } \\
\text { aspecto pero no } \\
\text { establece } \\
\text { ninguna } \\
\text { relación. }\end{array}$ & $\begin{array}{l}\text { Analiza y } \\
\text { estable } \\
\text { relaciones } \\
\text { razonadamente } \\
\text { (menos de dos) }\end{array}$ & $\begin{array}{l}\text { Analiza y } \\
\text { estable } \\
\text { relaciones } \\
\text { razonadamente } \\
\text { (dos o más) }\end{array}$ \\
\hline $\begin{array}{l}\text { Relaciona la seguridad de las } \\
\text { centrales con otros problemas } \\
\text { sociales (atentados, guerras, etc.). }\end{array}$ & No relaciona. & $\begin{array}{l}\text { Relaciona } \\
\text { superficialmente. }\end{array}$ & $\begin{array}{l}\text { Relaciona de } \\
\text { forma razonada } \\
\text { dos problemas }\end{array}$ & $\begin{array}{l}\text { Relaciona de } \\
\text { forma razonada } \\
\text { más de dos } \\
\text { problemas }\end{array}$ \\
\hline
\end{tabular}

Tabla 11. Rúbrica para “Centrales Nucleares Avanzadas” - Nivel de dominio II-

\begin{tabular}{|l|l|l|l|l|}
\hline Indicador No & $\begin{array}{l}\text { D. En desarrollo } \\
\text { alcanzado }\end{array}$ & $\begin{array}{l}\text { B. } \\
\text { /adecuado }\end{array}$ & $\begin{array}{l}\text { A. Excelente / } \\
\text { Ejemplar }\end{array}$ \\
\hline $\begin{array}{l}\text { Argumenta la necesidad de las } \\
\text { centrales nucleares en la sociedad del } \\
\text { futuro (a 20 años vista). Distingue la } \\
\text { tecnología madura de la no } \\
\text { convencional. }\end{array}$ & $\begin{array}{l}\text { No argumenta } \\
\text { ni distingue. }\end{array}$ & $\begin{array}{l}\text { Establece algún } \\
\text { argumento, pero } \\
\text { no lo defiende } \\
\text { adecuadamente. }\end{array}$ & $\begin{array}{l}\text { Establece } \\
\text { argumentos pero } \\
\text { no distingue } \\
\text { grado } \\
\text { madurez. }\end{array}$ & $\begin{array}{l}\text { Establece } \\
\text { argumentos } \\
\text { distingue } \\
\text { de madurez. }\end{array}$ \\
\hline $\begin{array}{l}\text { Prioriza las tecnologías nuevas que } \\
\text { pueden sustituir a las actuales } \\
\text { centrales nucleares y razona los pros } \\
\text { y contras de cada uno en el ámbito } \\
\text { del problema energético. }\end{array}$ & $\begin{array}{l}\text { No prioriza ni } \\
\text { razona. }\end{array}$ & $\begin{array}{l}\text { Prioriza, pero } \\
\text { no razona. }\end{array}$ & $\begin{array}{l}\text { Prioriza y razona } \\
\text { pero } \\
\text { superficialmente. }\end{array}$ & $\begin{array}{l}\text { Prioriza } \\
\text { razona } \\
\text { argumentos } \\
\text { válidos. }\end{array}$ \\
dando \\
\end{tabular}

Del trabajo realizado, se ha conseguido que los diferentes profesores de las asignaturas consideradas tengan una visión uniforme de lo que se pretende evaluar con cada una de las competencias transversales, mediante la realización de reuniones entre los profesores y establecimiento de las rúbricas más coordinadas en cada una de las competencias de las diferentes asignaturas involucradas en este trabajo. Además, con la definición y el 
desarrollo de una actividad, desde el punto de vista de las diferentes asignaturas, se facilita que el alumno vea un mismo problema desde ámbitos diferentes, lo que favorece que adquiera de forma más coherente y con mayor facilidad las diferentes competencias transversales.

Aunque este trabajo ha marcado las bases para poder realizar una evaluación de las competencias transversales de las diferentes asignaturas del ámbito de la Ingeniería Nuclear de manera más uniforme, coherente y coordinada, se necesita un tiempo de implementación para poder identificar posibles puntos de mejora. Queda por tanto pendiente la evaluación final y realimentación por parte del profesorado respecto al grado de implantación y resultados de la metodología planteada.

\section{Conclusiones}

La coordinación entre asignaturas es esencial para establecer las bases del trabajo competencial. El alumnado debe percibir una coherencia en el planteamiento, desarrollo y evaluación de las competencias transversales. En este trabajo se ha descrito el procedimiento mediante el cual se está realizando la coordinación de las competencias transversales en las diferentes asignaturas del ámbito de la ingeniería nuclear del Grado de Ingeniero de la Energía, en concreto con la competencia de "Conocimiento de problemas contemporáneos” como ejemplo de aplicación sin pérdida de generalidad. La coordinación debe establecer las bases de lo que los diferentes profesores van a transmitir a los alumnos y para ello lo primero realizado es el consenso en la definición de las diferentes competencias así como cuál debe ser el perfil del egresado en materia de ingeniería nuclear y se ha procedido a diseñar actividades al efecto con sus correspondientes rúbricas. Se ha intentado reforzar el mensaje trabajando una misma actividad con enfoques diferentes según la asignatura y estableciendo indicadores fácilmente medibles y transferibles entre asignaturas según el nivel de dominio. Dentro del último objetivo de la innovación, el trabajo requiere un proceso de mejora continua para subsanar las deficiencias que se detectan conforme se pone en práctica el proyecto. Como punto a destacar también por las dificultades que entraña es la conocida "libertad de cátedra" que algunos profesores alegan para evitar quedar condicionados en su materia por este tipo de coordinaciones.

Adicionalmente, se han obtenido similares productos dentro del proyecto de innovación para el resto de competencias dentro del ámbito de la ingeniería nuclear en el Grado de Ingeniero de la Energía pero cuya consecución y desarrollo es similar al analizado

\section{Agradecimientos}

Este trabajo se ha realizado en el marco del proyecto Proyecto de Innovación y Mejora Educativa, PIME Curso 2017-2018 "Coordinación de competencias transversales en asignaturas de ámbito nuclear en el Grado de Ingeniero de la Energía”, Referencia B07, del Vicerrectorado de Estudios, Calidad y Acreditación de la Universitat Politècnica de València.

(c) ) EY-NC-ND 2018, Universitat Politècnica de València

Congreso IN-RED (2018) 


\section{Referencias}

BERBEGAL et al "El portafolio digital de estudiantes como herramienta de evaluación de competencias", Red de Evaluación Alternativa en la Universidad http://www.unizar.es/real.

GALLARDO, S., CARLOS, S. (2015), "Desarrollo de la competencia transversal de "Análisis y Resolución de Problemas" en la asignatura Centrales Nucleares Avanzadas", $I N-R E D$, Valencia, Universitat Politècnica de València.

GALVÁN C. et al "Desarrollo de competencias transversales en entornos personales de aprendizaje en Educación Superior. Nuevas funciones de un sistema integrado de portafolios digitales”, III Congreso Internacional sobre Aprendizaje, Innovación y Competitividad (CINAIC 2015), Madrid, 2015.

OCHOA A. et al "Competencias en el contexto del Proyecto Mentor de una Escuela de Ingeniería, III Congreso Internacional sobre Aprendizaje, Innovación y Competitividad (CINAIC 2015), Madrid, 2015.

RODRÍGUEZ M.J. et al "Formación transversal de los estudiantes del Grado de Farmacia de la Universidad de Barcelona en un entorno de buenas prácticas", III Congreso Internacional sobre Aprendizaje, Innovación y Competitividad (CINAIC 2015), Madrid, 2015.

SANZ DE ACEO, M. L. (2010), "Competencias cognitivas en Educación Superior”, Narcea, Madrid.

VILLA, A. y POBLETE, M. (2007), “Aprendizaje basado en competencias. Una propuesta para la evaluación de las competencias genéricas”, Universidad de Deusto, Bilbao

UNIVERSITAT POLITÈCNICA DE VALÈNCIA, (2015a). "Presentación de Competencias Transversales UPV CT-10. Conocimiento de problemas contemporáneos”.

UNIVERSITAT POLITÈCNICA DE VALÈNCIA, (2015b). "Rúbrica UPV CT-10. Conocimiento de problemas contemporáneos”.

VILLANUEVA, J.F. et al. (2016), "Integración de la competencia transversal de "conocimiento de problemas contemporáneos" en Asignaturas de Grado", IN-RED, Valencia, Universitat Politècnica de València. 